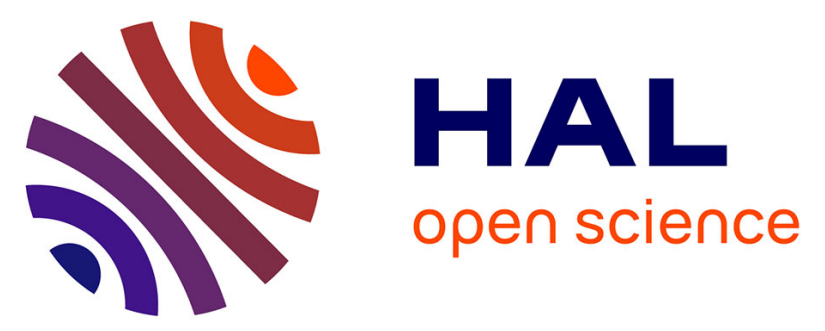

\title{
Dislocation Core Structure at Finite Temperature Inferred by Molecular Dynamics Simulations for 1,3,5-Triamino-2,4,6- trinitrobenzene Single Crystal
}

Paul Lafourcade, Christophe Denoual, Jean-Bernard Maillet

\section{- To cite this version:}

Paul Lafourcade, Christophe Denoual, Jean-Bernard Maillet. Dislocation Core Structure at Finite Temperature Inferred by Molecular Dynamics Simulations for 1,3,5-Triamino-2,4,6- trinitrobenzene Single Crystal. Journal of Physical Chemistry C, 2017, 121 (13), pp.7442-7449. 10.1021/acs.jpcc.6b11576 . hal-01676309

\section{HAL Id: hal-01676309 \\ https://hal.science/hal-01676309}

Submitted on 5 Jan 2018

HAL is a multi-disciplinary open access archive for the deposit and dissemination of scientific research documents, whether they are published or not. The documents may come from teaching and research institutions in France or abroad, or from public or private research centers.
L'archive ouverte pluridisciplinaire HAL, est destinée au dépôt et à la diffusion de documents scientifiques de niveau recherche, publiés ou non, émanant des établissements d'enseignement et de recherche français ou étrangers, des laboratoires publics ou privés. 


\title{
Dislocation Core Structure at Finite Temperature Inferred by Molecular Dynamics Simulations for 1,3,5-Triamino-2,4,6- trinitrobenzene Single Crystal
}

\author{
Paul Lafourcade, ${ }^{\dagger, \ddagger 0}$ Christophe Denoual, ${ }^{* \dagger}$ and Jean-Bernard Maillet ${ }^{\dagger}$ \\ †Département de Physique Théorique et Appliquée, CEA-DAM IdF, F-91297 Arpajon, France \\ †Procédés et Ingénierie en Mécanique et Matériaux (PIMM), Arts et Métiers ParisTech, 151, boulevard de l'Hôpital, F-75013 Paris, \\ France
}

ABSTRACT: The dislocation core structures and elastic properties of the insensitive energetic molecular crystal 1,3,5-triamino-2,4,6trinitrobenzene (TATB) are investigated as a function of pressure and temperature. A new method is proposed to compute the generalized stacking fault surfaces (noted $\gamma$-surfaces) and the complete second-order elastic tensor at finite temperature through molecular dynamics (MD) simulations. The energy landscapes in the two glide planes are shown to be similar between 0 and $300 \mathrm{~K}$, thus leading to almost no modification on the dislocation evolution. A spreading of the dislocation cores over a hundred Burgers vectors is

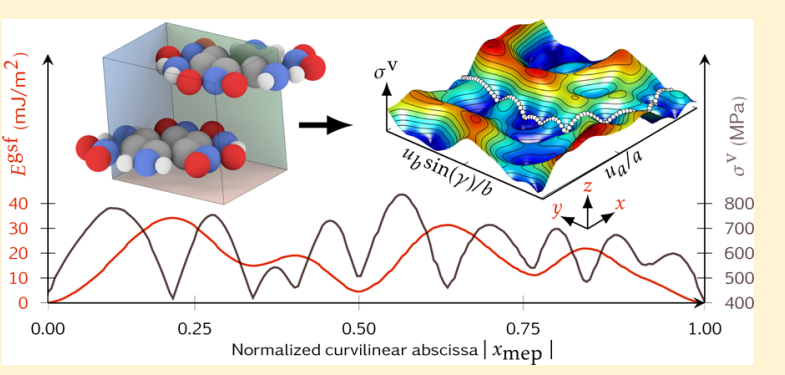
observed along the [100] and [010] directions for the edge and screw dislocations at 0 and $300 \mathrm{~K}$, showing that dislocations should exhibit a very low friction for these glide systems at ambient pressure. For pressures varying between 0 and $10 \mathrm{GPa}$, the $\gamma$ surfaces' energy barriers that drive the width of partial dislocations follow the increase of shear elastic constants within the considered glide planes, thus limiting the changes of the dislocation core structure.

\section{INTRODUCTION}

1,3,5-Triamino-2,4,6-trinitrobenzene (TATB) is a well-known highly insensitive energetic molecular crystal used in various explosive formulations that crystallizes in a triclinic cell of space group $P \overline{1}{ }^{1}$ It exhibits a very anisotropic thermo-mechanical and chemical behavior ${ }^{2-4}$ due to the arrangement in layers of the two $\mathrm{C}_{6} \mathrm{H}_{6} \mathrm{~N}_{6} \mathrm{O}_{6}$ molecules (see Figure 1). Indeed, the strong intramolecular hydrogen bonding between atoms of adjacent nitro and amino groups and the hydrogen bonding between molecules within the basal plane layers contrast with interplanar interactions, governed by weak van der Waals forces. These two types of interactions imply anisotropic thermal and mechanical properties, as suggested by estimations of the second order elastic tensor, ${ }^{2,5}$ which predict longitudinal celerity 20 times faster for the [100] and [010] directions compared to [001].

Despite numerous studies devoted to a better understanding of TATB behavior under moderate loadings, the mechanisms of its irreversible deformation still remain poorly understood. Due to the difficulties to obtain single crystals of compatible size with standard mechanical testing machines as well as security constraints, no experimental data is available on, for example, single TATB crystal under pure uniaxial tension. For these reasons, the numerical modeling of TATB crystal plays an essential part in studying its macroscopic behavior. For example, Mathew et al. ${ }^{6}$ and Kroonblawd et al. ${ }^{7}$ studied through molecular dynamics (MD) simulations its elasticplastic response under displacement-controlled nanoindenta-

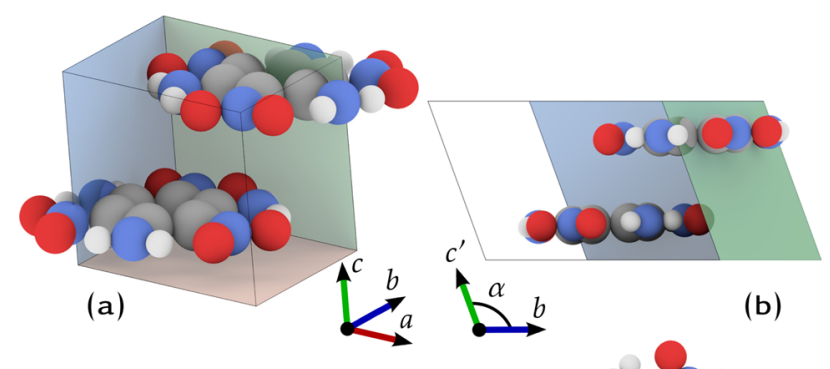

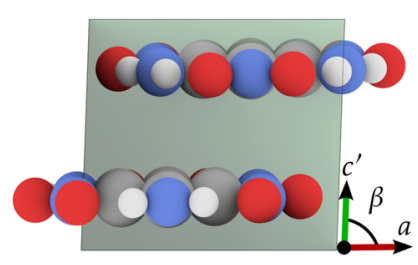

(c)

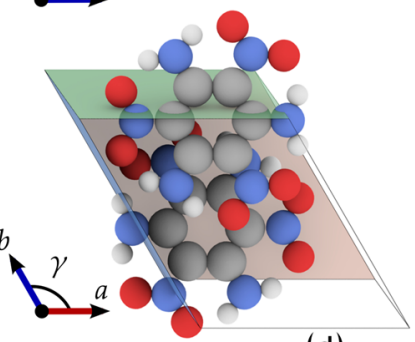

(d)
Figure 1. Crystal structure. TATB unit cell with lattice vectors $\mathbf{a}, \mathbf{b}$, and $\mathrm{c}$, and cell angles $\alpha, \beta$, and $\gamma$. (a) A 3D representation. (b) $x+$ view. (c) $y+$ view. (d) $z+$ view.

Received: November 17, 2016

Revised: February 10, 2017

Published: March 16, 2017 
tions and under shock. They reported that some studies ${ }^{8}$ have suggested the existence of high defect density in grown crystals of TATB and subtle structural phase transitions under hydrostatic compression. The generalized stacking fault energies within the two glide planes have been computed ${ }^{5}$ using all-atom simulations with a classical force field and suggested that easy dislocation glide could be present in TATB.

The sensitivity of energetic materials is related to their capacity to remain stable under mechanical and thermal loadings. Initiation of detonation in explosive crystals is believed to occur because of the formation of hot spots. ${ }^{6,9-11}$ The latter can be created by material defects and plastic deformation mechanisms that induce energy localization within the material. Various papers have suggested that void collapse, ${ }^{12,13}$ localized heating due to plastic deformation, ${ }^{9}$ and dislocation pile-up ${ }^{6}$ are some specific mechanisms that could involve initiation of detonation. Thus, a detailed modeling of plasticity is mandatory if one wants to understand the mechanisms of ignition.

Dislocations are known to be the main mechanism that control plasticity in crystalline materials but their existence in TATB has never been assessed. The definition of predictive MD potentials ${ }^{2,14}$ is the first step toward the prediction of dislocation core structures and their mobility. However, the unusual elastic anisotropy, as well as the very low energy along the gliding directions, impose one to consider possible large dislocation cores and thus huge (and expensive) simulation setup. To overcome this difficulty, a numerical method derived from the Peierls-Nabarro (PN) model $^{15}$ called PeierlsNabarro Galerkin (PNG, see refs 16 and 17) is considered. In this method, the dislocation core structure emerges through the competition of a potential energy (called the $\gamma$-surface) and the elastic energy. It has been used for numerous studies dedicated to the simulation of complex dislocation core structures for metals ${ }^{17}$ or earth mantle materials. ${ }^{18,19}$

For the latter studies, the PNG computations were performed at $0 \mathrm{~K}$ to provide an estimate of the so-called Peierls-stress, ${ }^{20}$ defined as the ultimate shear stress without thermal activation. Noting the important dependency of thermodynamical and mechanical properties with $T$ and keeping our focus on dislocation existence and core structure, we propose in the following a method to obtain, through a PNG calculation, an estimation of dislocation core structure under nonzero temperature. The understanding of plasticity mechanisms in TATB single crystal is essential to perform simulations of plastic deformations at nanometer scale as done by Koslowski et al., who studied ${ }^{21,22}$ the role of partial dislocations and stacking fault ribbons in 3D nanocrystalline materials depending on the grain size using a phase-field theory of dislocation dynamics. ${ }^{23}$

The methodology presented hereby is dedicated to the study of dislocations in TATB and their thermal and pressure dependence. $\mathrm{MD}$ simulations were used to provide generalized stacking fault energies and elastic constants, as input parameters of the PNG method. The second order elastic tensor and the $\gamma$ surfaces have been computed for several $(T, P)$ conditions in order to predict the structure of edge and screw dislocations along the two Burgers vectors corresponding to the lattice vectors $\mathbf{a}$ and $\mathbf{b}$. We then discuss the existence of dislocations and plastic behavior in the last section.

The paper is organized as follows. In the first section, results from MD simulations of TATB under various conditions and the methodology proposed to obtain $\gamma$-surfaces at finite temperature and second-order elastic tensor are presented. The second section is then dedicated to macroscopic simulations using the PNG model to study dislocation core structure in TATB. Structures for edge and screw dislocations along the principal directions of the two glide planes taken in consideration are investigated.

\section{MICROSCOPIC APPROACH}

2.1. MD Potentials, Structure, and EOS. All simulations were performed using the STAMP code. ${ }^{24}$ The nonpolarizable, $20 \%$ scaled-charges force field for TATB initially developed by Bedrov et al. ${ }^{2}$ was used with planar, symmetric rigid molecules. They predicted for the first time TATB elastic constants and crystal structure evolution in $T$ and $P$. This force field was modified by Kroonblawd and Sewell ${ }^{3,4}$ and used to investigate the thermal behavior of TATB and more recently to obtain the first generalized stacking fault surface and to explore the mechanisms of deformation under nanoindentation. ${ }^{5}$

Instead of the Ewald summation for the computation of longrange electrostatic interactions, the Reaction Field ${ }^{25-27}$ approximation with a $13 \AA$ cutoff and 100.0 for the dielectric constant $\varepsilon$ was used. A Langevin thermostat was used with a damping constant set to $1.0 \mathrm{ps}$. The time step for integrating the equations of motion was set to 1.0 fs for both isochoricisoergic (NVE) and isochoric-isothermal (NVT) simulations, small enough regarding the fact that simulations are performed with rigid molecules. Repulsion, dispersion, and short-range electrostatic interactions were computed with a cutoff distance of $13 \AA$. All intramolecular nonbonded interactions were excluded in the implementation of the force field. Finally, the rigid body dynamics is computed by discretization of equations of motion following a Velocity-Verlet scheme. ${ }^{28}$

Discussion on the Choice of Planar and Rigid Molecules. In a recent work, ${ }^{29}$ the contribution of molecular flexibility to the elastic-plastic properties of molecular crystal $\alpha$-RDX has been studied. The authors evaluate crystal-scale parameters as the elastic constants, lattice parameters, thermal expansion coefficients, stacking fault energies, and the Peierls-stress for the motion of a dislocation. They show that the mechanical properties of the $\alpha$-phase of RDX can be strongly affected by the flexibility of the constituent molecules. In our case, the ring of TATB molecules is found to be highly stable, giving the molecules an almost planar geometry. Because TATB molecules do not exhibit consequent flexible behavior compared to the RDX ones, we have chosen to perform our $\mathrm{MD}$ simulations with perfectly planar, symmetric, and rigid molecules.

Cell Parameters Evolution in $T$ and $P$. Molecules orientations and center of mass (COM) positions were obtained through Parrinello-Rahman ${ }^{30,31}$ simulations for different temperatures and pressures. $P$ and $T$ coupling constants were set to $0.1 \mathrm{ps}$ and $1.0 \mathrm{fs}$, respectively. Starting with the experimental structure ${ }^{1}$ to initiate Parrinello-Rahman simulations, a $3 \mathrm{D}$-periodic $5 \boldsymbol{a} \times 5 \boldsymbol{b} \times 7 \boldsymbol{c}$ simulation cell containing 350 molecules was created. TATB cell parameters for each $(T, P)$ state were obtained by averaging the values over the final 150 ps of a 250 ps Parrinello-Rahman simulation. The averaged lattice parameters are given in Table 1. Uncertainties correspond to one standard deviation of the mean and were evaluated following the block averages method. ${ }^{32}$

It is to be noted that angle $\alpha, \beta$, and lattice parameter $c$ deviate from experiment ${ }^{1}$ for a few percent and this is probably 
Table 1. Unit Cell Parameters from MD Parrinello-Rahman Simulations and Experimental Values at Ambient Conditions

\begin{tabular}{lcccc} 
& experiment & this work & deviation (\%) & uncertainties $(\sigma)$ \\
$a(\AA)$ & 9.010 & 9.009 & -0.01 & $1.2 \times 10^{-3}$ \\
$b(\AA)$ & 9.028 & 9.041 & 0.14 & $9.1 \times 10^{-4}$ \\
$c(\AA)$ & 6.812 & 6.570 & -3.55 & $5.9 \times 10^{-4}$ \\
$\alpha(\mathrm{deg})$ & 108.58 & 103.34 & -4.83 & $7.8 \times 10^{-3}$ \\
$\beta(\mathrm{deg})$ & 91.82 & 88.34 & -3.79 & $1.5 \times 10^{-2}$ \\
$\gamma(\mathrm{deg})$ & 119.97 & 120.70 & +0.61 & $6.8 \times 10^{-3}$ \\
\hline
\end{tabular}

due to the planeness and rigidity imposed to the molecules as suggested by $\mathrm{Pal}$ and Picu recently. ${ }^{29}$

In Figure 2, cell lengths and cell angles evolution is represented with $P$ and also the equation of state (EOS) of
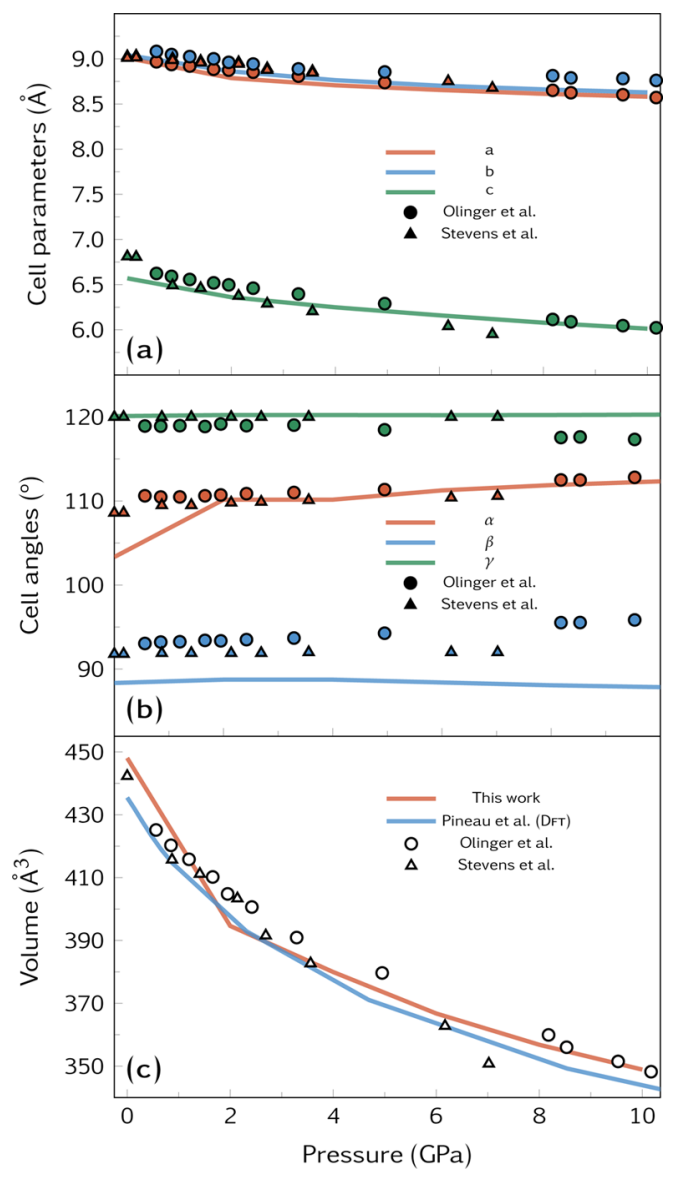

Figure 2. Pressure dependence of TATB cell lengths (a), cell angles (b) at $300 \mathrm{~K}$, and cold curve (c) from MD simulations compared with experiment and density functional theory data.

TATB. These results are compared to the two experiments driven by Stevens et al. ${ }^{33}$ and by Olinger and $\mathrm{Cady}^{34}$, and with a density functional theory study of Pineau et al. ${ }^{35}$ for the EOS. Our results are found to be in a good agreement with literature even though little differences can be observed on the $\beta$-angle that progresses under $90^{\circ}$ contrary to experiments and on the $c$ length which is $4 \%$ smaller, probably due to the planar symmetry imposed to the TATB molecules. Nevertheless, it can be observed that the agreement between simulations and experiments seems to improve for increasing pressures.

Quasi-linear coefficients of thermal expansion (CTE) of 1.95 $\times 10^{-4}, 1.94 \times 10^{-4}$, and $3.60 \times 10^{-4} \AA / \mathrm{K}$ were deduced from simulation results for $\mathbf{a}, \mathbf{b}$, and $\mathbf{c}$ directions, respectively. These values are consistent with the crystal structure but are shown to be quite different from those obtained by Bedrov, ${ }^{2}$ particularly for the one in the $\mathbf{c}$ direction. Yet, experimental data ${ }^{33}$ for CTE allow us to observe that our simulation underestimates the values for the $\mathbf{b}$ and $\mathbf{c}$ directions while it overestimates the one in the a direction. In their recent work, ${ }^{29} \mathrm{Pal}$ and Picu demonstrate that the CTE can strongly depend on molecular structure and flexibility. In our case, the approximation on the planeness of TATB molecules and their rigidity (by constraining dihedral and angular flexibility of the ring, amino, and nitro groups) may affect significantly the CTE. The principal aim of this work is to compute elastic constants and $\gamma$-surfaces at the microscopic scale in order to calculate dislocation core structures at an upper scale, in which the CTE do not play any role. Thus, the small differences in the crystal structure compared to the ones published in the literature ${ }^{1,2,5}$ may not have a strong influence on the general conclusions on the dislocation core structures of TATB, regarding the similarities between elastic constants and $\gamma$-surfaces obtained in this work compared to the results of the literature.

2.2. Elastic Tensor in Pressure and Temperature. The complete second-order stiffness tensor $\mathbf{C}$ was obtained through a large range of $T$ and $P$. The coefficients were calculated by fitting a second-order polynomial on a set of elastic-energy versus strain equations

$$
\begin{aligned}
E(V, \varepsilon)=E\left(V_{0}, 0\right) & +V_{0} \sum_{i, j=1}^{3}\left(\sigma_{i j}\right)_{0} \varepsilon_{i j} \\
& +\frac{V_{0}}{2} \sum_{i, j=1}^{3} C_{i j k l} \varepsilon_{i j} \varepsilon_{k l}+\ldots
\end{aligned}
$$

where Hooke's law expression is contained in the third term, expressing the second-order Cauchy stress tensor $\sigma$ as a function of the linearized strains tensor $\varepsilon$ and $\mathbf{C}$ with $V_{0}$ the cell volume. These elastic-energy versus strain curves were obtained through $\mathrm{MD}$ simulations by successive homogeneous deformations of a $3 \mathrm{D}$-periodic $20 \mathrm{a} \times 20 \mathrm{~b} \times 20 \mathrm{c}$ simulation cell. No relaxation was performed after deformation, and therefore the instantaneous elastic response is measured. This measure is however comparable to the relaxed response because the prescribed deformations are too small to induce an important atomic rearrangement with the noticeable advantage to get rid of the noise that considerably hinders the measure of an accurate energy variation. Moreover, a system of 16000 molecules was taken to improve the ensemble average and thus the accuracy of the measure. In eq $1, E$ stands for potential energy. The system is considered to be isentropic because no relaxation at all is performed after the strain is applied, that is, the atomic positions remain unchanged. What is computed is consequently the isentropic elastic constants $C_{i j}^{S}$, that is, the stiffness implied in acoustic waves for large wavelengths (see Wallace $\left.{ }^{36}\right)$. For practical reasons, the notation $C_{i j}$ is used in the remaining of this paper.

In order to obtain the stiffness matrix for each couple of $(T$, $P)$, a new simulation cell was created for each condition. Starting with the cell parameters obtained with the ParrinelloRahman method, a 3D-periodic simulation cell of $20 \mathbf{a} \times 20 \mathbf{b} \times$ 20c was considered and thermalized in the NVT ensemble during $250 \mathrm{ps}$. The procedure to compute the elastic constants at finite temperature is the following: once the supercell is 
thermalized, we randomly pick about 50 stable configurations among the final 100 ps of the NVT simulation. We then strain these configurations to obtain the elastic-energy versus strain curves (around 50 for each stiffness tensor component) that are gathered and averaged in order to obtain a single "mean" energy curve for each elastic constant, much more representative of the supercell elastic state due to the applied strain. The strains were prescribed to the supercell while maintaining the center of mass (COM) of each molecule at the same position in terms of fractional coordinates. As TATB crystallizes in a triclinic structure, 21 elastic coefficients are needed to obtain the complete stiffness matrix. Therefore, 21 homogeneous deformation types had to be applied for the homogeneous deformation method. The six diagonal components of $\mathbf{C}$ were determined by applying pure stretch or pure shear and the nondiagonal components by imposing a combination of both. The elastic energy versus strain curves were determined for strains ranging between $-0.5 \%$ and $+0.5 \%$ with a $0.1 \%$ step. Two errors have to be considered here. The first comes from the average of multiple elastic-energy versus density curves and is lower than $2 \%$ while the second is due to the fitting and is always lower than $1.2 \%$.

Results for $300 \mathrm{~K}$ and $0 \mathrm{GPa}$. The complete second-order elastic tensor at ambient conditions, which clearly reflects the anisotropic behavior of TATB, is given in the Supporting Information. This anisotropy is consistent with the crystal structure because deformations along $\mathbf{a}$ and $\mathbf{b}$ directions involve strong hydrogen bonding whereas only weak van der Waals forces act along the $\mathrm{c}$ direction. Analogous reasoning can be made concerning the shear coefficients $\left(C_{44}, C_{55}\right.$, and $\left.C_{66}\right)$ and the dilatation ones $\left(C_{12}, C_{13}\right.$, and $\left.C_{23}\right)$, which are in agreement with our measures that give $C_{66} \gg C_{44}, C_{55}$ and $C_{12} \gg C_{13}, C_{23}$. Furthermore, elastic constants have been computed by Mathew et al. $^{5}$ and Bedrov et al. ${ }^{2}$ through hybrid MD-Monte Carlo simulations using the Parrinello-Rahman strain fluctuation formula. ${ }^{37}$ Overall, the same anisotropy was observed and the results are in good agreement even if an higher value for $C_{33}$ was obtained in our case.

Pressure Dependence of the Elastic Coefficients. In Figure 3 shows the dependence of the elastic coefficients with pressure. The ratio between the $C_{i j}$ at $10 \mathrm{GPa}$ and the $C_{i j}$ at $0 \mathrm{GPa}$ remains constant, thus leading to the same elastic anisotropy. The complete elastic tensors over the $P$ and $T$ total ranges investigated are provided in the Supporting Information.

2.3. $\gamma$-Surfaces and Minimum Energy Paths. The $\gamma$ surface is a potential energy calculated by prescribing a gliding $f$ along the dense plane of a perfect crystal. In order to minimize the energy, an additional relaxation is usually considered in the direction perpendicular to the gliding surface (see ref 5 for a $\gamma$ surface in TATB at $0 \mathrm{~K}$ ). Because of this relaxation, and consistently with common practices that use $\gamma$-surface to calculate dislocation core structure without thermal activation, this potential is calculated at $0 \mathrm{~K}$ only.

Because dislocation structure calculated at $0 \mathrm{~K}$ could differ from the one obtained in temperature, we present in the following a method to define a $\gamma$-surface that can be used to calculate dislocations core structure at finite temperature.

A measure at finite temperature requires an average over an ensemble of configurations representatives of the state of the system. However, for a $\gamma$-surface computation the additional constraint on particle locations hinders dynamical evolution, now restricted to the direction perpendicular to the glide plane. To overcome this limitation, we propose to perform the

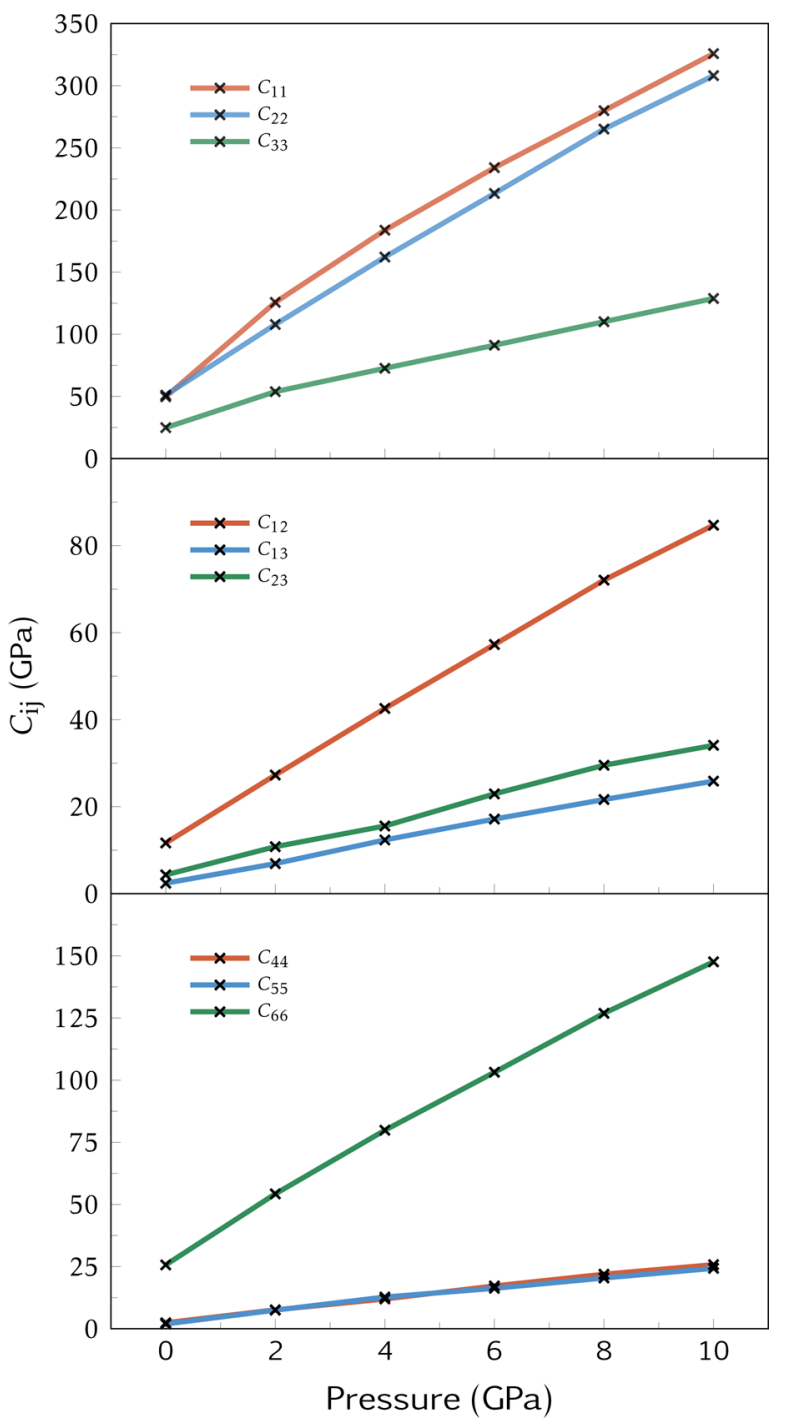

Figure 3. Evolution of the nine orthotropics stiffness coefficients of TATB crystal at ambient temperature.

sampling over the equilibrium state at finite temperature before deformation. The deformation is then instantaneously applied to this set of configurations and the final energy is computed as the average of the energies of the deformed systems over this ensemble. Assuming ergodicity, this ensemble of configurations is equivalent to a large system. A crystal of $20 a \times 20 b \times 20 c$ is then considered.

A nonorthogonal basis $(a, b, c)$ defines the crystal lattice. A second orthogonal basis $(x, y, z)$ is defined by using the convention such that $a$ is aligned with $x, b$ is lying in the $(x, y)$ plane and $c$ is oriented in the upper Cartesian half-space and makes angles of $\beta$ and $\alpha$ with $a$ and $b$, respectively. The TATB cell contains two different slip planes $P 1$ and $P 2$, and thus two $\gamma$-surfaces have to be computed.

For each couple of parameters $(T, P)$, a $3 D$-periodic crystal is built from the cell parameters obtained with the ParrinelloRahman simulation and equilibrated in the NVT ensemble for about 100 ps. The crystal is then split at $P 1$ location (respectively P2) and an instantaneous glide in the $(x, y)$ plane is applied to the upper part. In order to compute the energy, the periodicity along the $z$-direction is removed because the displacement breaks the system symmetry. 


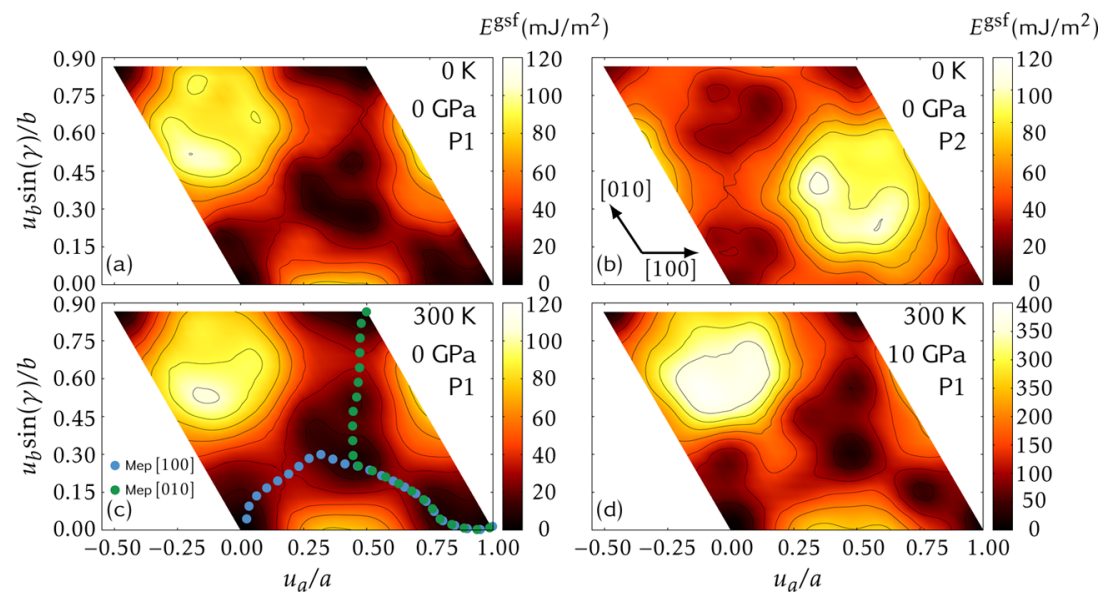

Figure 4. $\gamma$-surfaces for TATB at $0 \mathrm{~K}$ and $0 \mathrm{GPa}$ for $P 1$ (a) and $P 2(\mathrm{~b})$ planes. The $300 \mathrm{~K} \mathrm{P1}$ plane $\gamma$-surfaces at $0 \mathrm{GPa}(\mathrm{c})$ and $10 \mathrm{GPa}(\mathrm{d})$. MEP along $[100]$ and $[010]$ directions obtained with the string method are represented on the bottom left image.

It is worth mentioning that such "in temperature" $\gamma$-surface is not periodic, that is, the energy after a displacement $a$ could be different from the energy without displacement, as each molecule has a different position and orientation. In order to minimize this discontinuity, we chose to compute the energy of 16 different configurations corresponding to 16 lattices centered on the stable configuration $([-2 a,+2 a],[-2 b$, $+2 b]$ ). This additional average decreases the discontinuity by 2 orders of magnitude, down to $0.5 \%$.

$\gamma$-surfaces for the basal plane of TATB at $0 \mathrm{~K}$, ambient conditions, and for five equally spaced pressures were computed. Because a glide of $a[100], b[010]$ or the transverse direction $(\alpha[110])$ restores the initial structure, it can be interesting to investigate the thermal and pressure effect over the energetic barriers for these directions. The $0 \mathrm{GPa} \gamma$-surfaces computed with rigid molecules for the $P 1$ and $P 2$ basal plane at $0 \mathrm{~K}$ are represented in Figure 4 and are comparable to the ones recenlty obtained by Mathew et al. ${ }^{5}$ In Figure 4, different $\gamma$ surfaces are represented. The comparison of the (0 GPa, 300 $\mathrm{K})$ case with the $(0 \mathrm{GPa}, 0 \mathrm{~K})$ suggests that the main effect of temperature is a slight smoothing of intermediate minima and energetic barriers. Because the lattice parameters are not subjected to noticeable changes when performing $\mathrm{MD}$ Parrinello-Rahman simulations at $300 \mathrm{~K}$, the interplanar distance between the glide planes remains almost identical, leading to no modification of the $\gamma$-surfaces shape.

The $\gamma$-surfaces shape (see Figure 4) does not experience a strong evolution under pressure, consistently with the high stability of the triclinic structure of TATB. This can be checked on the minimum energy path (MEP) plots computed for a gliding of $a[100]$ and $b[010]$ by using the string method ${ }^{38}$ for different pressures (see Figure 5). In these MEP graphs, the energy values have been normalized by an estimation of the average shear constant within the glide plane (estimated by the Birch coefficients (see eq 3 , below) $\left(B_{44}+B_{55}\right) / 2$ ) in order to measure the equilibrium between elastic and inelastic energy. By shifting the different normalized energy curves, one can notice that the energy barriers (i.e., the difference between the stable and unstable stacking fault energies) evolution follows the stiffness, which emphasizes that the anisotropy is not enhanced as well as the partial dislocations core width.

Existence of multiple minima along the MEP that are not located along the straight glide line is a strong indication that the dislocation core is composed of several partial dislocations

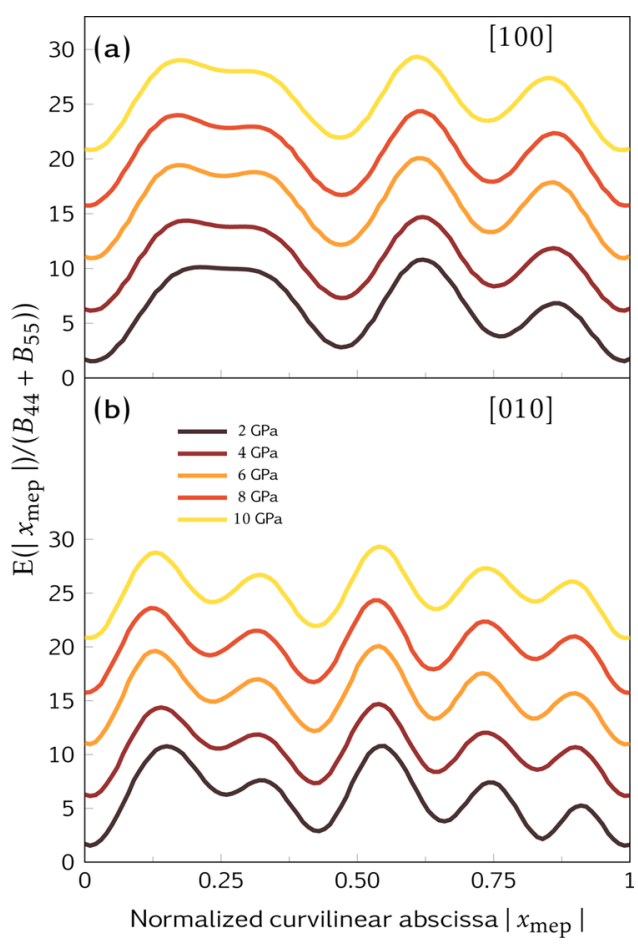

Figure 5. Energies along MEP normalized by shear elastic coefficients along [100] (a) and [010] (b) for the studied range of pressures. Curves have been shifted to highlight their similar evolution.

with partial Burgers vector noncolinear to the dislocation Burgers vector. In Figure 5, the stable stacking fault energies are slightly increasing with pressure, which will have an impact on the partial dislocations equilibrium splitting. This point is further discussed in the next section dedicated to the modeling of dislocation core structure by the PNG model. It is interesting to differentiate the $\gamma$-surfaces with respect to the twodimensional vector $\mathbf{f}$ lying in the plane in order to obtain the surface density of forces $\sigma n$. It represents an estimation of the necessary von Mises shear stress required to activate homogeneous gliding, that is, without moving dislocations. Along the two MEPS the maximal von Mises stress approaches $700-800 \mathrm{MPa}$ at ambient state (0 GPa, $300 \mathrm{~K})$, which is comparable to the ratio $C_{55} / 6$. 


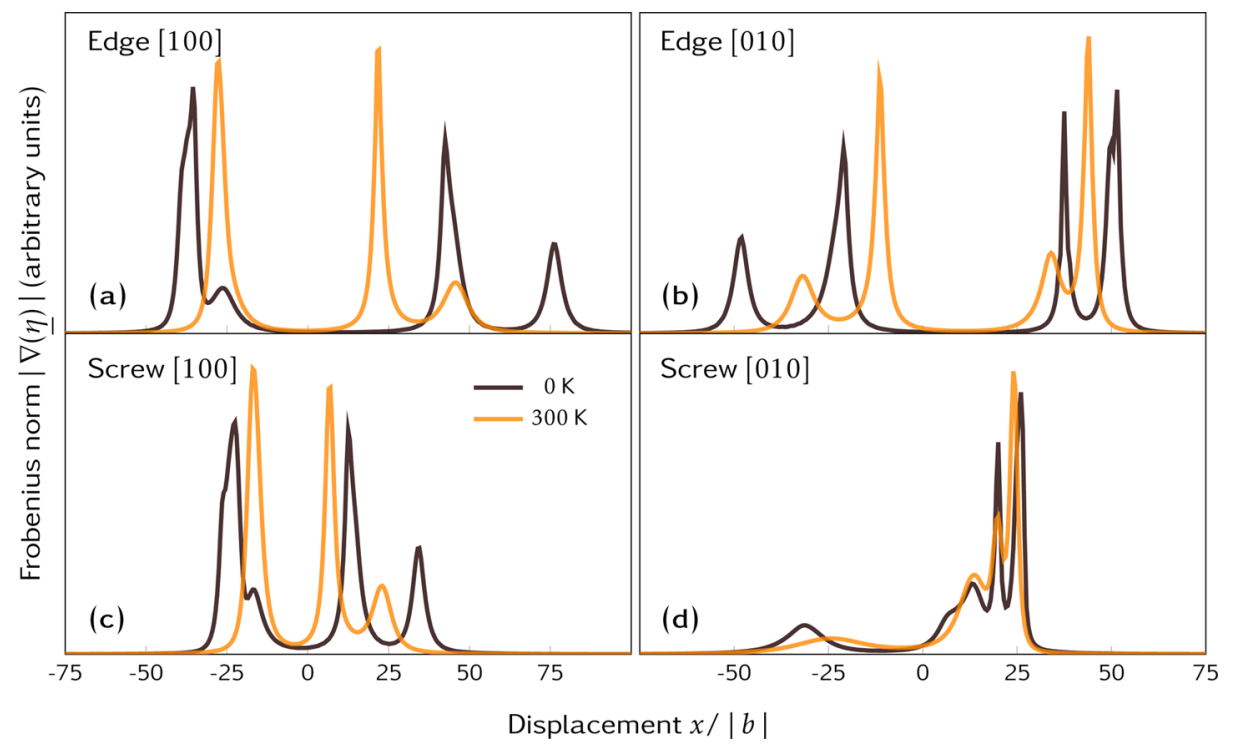

Figure 6. Frobenius norm of $|\nabla \eta|$ through the dislocation cores for edge dislocation along [100] (a) and [010] (b) directions and for screw dislocations along $[100](\mathrm{c})$ and $[010](\mathrm{d})$ directions.

We have considered planar and rigid molecules for our MD simulations in order to compute elastic constants and $\gamma$ surfaces. Results presented in the literature for both stiffness tensor $^{2,5}$ and $\gamma$-surfaces ${ }^{5}$ have been obtained through MD simulations with flexible molecules. Because our results with rigid molecules are similar to those obtained previously with flexible molecules both for $\gamma$-surfaces and elasticity, we consider our hypothesis to be justified, thus allowing to use these results as input of an upper-scale simulation such as a dislocation core structure computation.

\section{DISLOCATION CORE STRUCTURE}

The Peierls-Nabarro-Galerkin method is a technique developed to calculate complex dislocation core, e.g., when the core is spread along different nonparallel glide plane, ${ }^{17}$ with very complex crystallographic structure, ${ }^{18}$ or submitted to a dynamic loading. ${ }^{17}$ These calculations are performed without temperature, which is consistent with the measure, by using the PNG, of the dislocation activation stress, only defined at $0 \mathrm{~K}$.

One can notice however that the vibration frequency of a dislocation is several orders of magnitude lower than the frequency of atomic vibrations. A time-scale separation could thus be introduced between the dislocation core structure and the molecules positions and orientations at equilibrium. This opens the way to the definition of $\gamma$-surfaces at finite temperature that can be used in a PNG dislocation core structure calculation.

3.1. Peierls-Nabarro-Galerkin Model. The PNG model associates a generalized Peierls-Nabarro (PN) model with an element-free Galerkin method, a method comparable to finiteelement techniques. The classical PN model assists in the minimization of the total energy functional split in an elastic part and an interplanar potential computed from the $\gamma$-surface, leading to the dislocation core structure. The interplanar potential for the PNG method is the $\gamma$-surface from which the elastic part has been removed. Two distinct fields are introduced: a three-dimensional displacement field $\mathbf{u}$ and a two-dimensional one $\eta$ lying in the plane of the $\gamma$-surface. A solution (i.e., a dislocation core structure) is finally obtained through the energy minimization with respect to $\mathbf{u}$ and $\eta$ of the energy functional $\mathcal{E}$ :

$$
\mathcal{E}=\underbrace{\frac{1}{2} \int_{V}\left(\nabla \mathbf{u}-\eta \varepsilon^{0}\right) \mathbf{B}\left(\nabla \mathbf{u}-\eta \varepsilon^{0}\right) \mathrm{d} \mathbf{r}}_{\text {elastic energy } \mathrm{E}^{\mathrm{e}}}+\underbrace{\int_{S} E^{\text {isf }}[\eta] \mathrm{d} \mathbf{r}}_{\text {inelastic energy }}
$$

where $E^{\mathrm{e}}$ and $E^{\text {isf }}$ are the elastic energy and the inelastic stacking fault energy (also called the "crystalline energy"), respectively, $\mathbf{B}$ is the Birch coefficients (or stress/strain) tensor ${ }^{39}$ which is deduced from the elastic tensor $\mathbf{C}$ with the following formulas ${ }^{36,40}$

$$
\mathbf{B}_{i j k l}=\mathbf{C}_{i j k l}-P\left(\delta_{j l} \delta_{i k}+\delta_{i l} \delta_{j k}-\delta_{i j} \delta_{k l}\right)
$$

where $P$ is the pressure ( $P>0$ for compression).

The determination of $E^{\text {isf }}$ depends on material properties and governs the inelastic behavior of the dislocation core. This energy is built such that the computed total energy from the continuum fields exactly equals $E^{\mathrm{gsf}}$, leading to a minimization relation connecting these two energies. ${ }^{16}$ Thus, the only input here are the generalized stacking fault energies $E^{\text {gsf }}$ obtained by independent $\mathrm{MD}$ calculations of block-sliding configurations, and the complete second order stiffness tensor $\mathbf{C}$ obtained by $\mathrm{MD}$ calculations of homogeneous deformations. An interpolation is performed on the $\gamma$-surfaces (inferred by $\mathrm{MD}$ simulations) in order to obtain a much more refined representation of $E^{\mathrm{gsf}}$. The interpolation error has been set to $0.5 \mathrm{~mJ} \cdot \mathrm{m}^{-2}$, leading to a very accurate interpolation of the $\gamma$ surface.

3.2. Dislocation Core in TATB. For all calculations, a $2 \mathrm{D}$ mesh is considered with dimensions 1024 TATB unit cells in the $x$-direction (that corresponds to crystallographic direction a), 256 unit cells in the direction perpendicular to the glide plane (direction $z$ ), and one unit cell in the $y$-direction. The nodal resolution is two Element Galerkin nodes per lattice edge in $x$ - and $y$-directions (i.e., $2048 \times 512$ nodes are considered). Last top and bottom nodes rows have a displacement constrained in the $y$-direction and have no constraint in the $x$-direction. A discrete dislocation is introduced at the volume 
center and its structure relaxed to its minimal energy following the procedure proposed by Denoual. ${ }^{17}$ The dislocation structure is then analyzed in terms of the spreading of the $\eta$ field representing the inelastic part of the disregistry. ${ }^{16}$

Results for the dislocation core at $0 \mathrm{~K}$ and ambient temperature for edge and screw dislocations along $a[100](001)$ and $b[010](001)$ directions for the two glide planes are first presented. Then, only the $P 1$ plane is discussed to illustrate the impact of pressure on edge and screw dislocations along $a$ and $b$, because the behavior in the two glide planes $P 1$ and $P 2$ is very similar regarding their energy landscape and dissociation paths.

Ambient Pressure [100](001) Dislocations. The dislocation core structure can be determined by analyzing the Frobenius norm of the gradient of $\eta,|\nabla \eta(x)|$ (in Figure 6), which represent the density of Burgers vector. Thus, each peak of $|\nabla \eta(x)|$ represents a partial dislocation, the width of which is characterized by the full width at half-maximun (fwhm). In all simulations, dislocations appear to split into several partials with fwhm around 10 Burgers vectors.

First, it appears that the [100] edge dislocations gliding leads to a very complex core structure that spreads out over around 125 Burgers vectors at $0 \mathrm{~K}$. The [100] screw dislocations also exhibit a complex (but thinner) core, spread over 75 Burgers vectors. Not only are those widths several orders of magnitude higher than the one that could lead to a measurable dislocation friction but they also reveal that dislocations in TATB cannot be considered as discrete lines but rather as ribbon of stacking faults.

The second set of simulations shows that an increase of $T$ leads to a reduction of the spreading length for both edge and screw dislocations. Indeed, values of 75 and 50 Burgers vectors, respectively, are found, still too high for considering dislocations as discrete objects.

Ambient Pressure [010](001) Dislocations. The [010] and [100] dislocations have similar Burgers vector lengths and energy barriers. A comparable behavior concerning the dislocation core spreading within the glide plane is then expected. Here as well, screw dislocations spread over a smaller distance than edge ones.

The results for the $P 2$ plane depict similar trends. For both edge and screw dislocations, for the two glide directions and the two glide planes, dislocations under ambient pressure have no localized core, and temperature sligthly reduces the core spreading about approximatively 25-30\%.

Effect of Pressure on the Equilibrium Splitting of Partial Dislocations. Because the PNG model consists in finding the equilibrium between elastic and stacking fault energy, one can anticipate how the dislocation core structure is affected by the pressure. All dislocations seem to split into several partials.

The results (presented in the Supporting Information) show that the splitting between partials increases with pressure of about $50 \%$ between 0 and $10 \mathrm{GPa}$. This is consistent with the trend for dislocation partials to follow the evolution of $G \mathbf{b}_{1} \mathbf{b}_{2}$ / $\gamma_{\mathrm{SF}}$ (with $G$ the average modulus, $\mathbf{b}_{1}$ and $\mathbf{b}_{2}$ the norm of the two Burgers vectors, and $\gamma_{\mathrm{SF}}$ the stacking fault energy ${ }^{41}$ ).

\section{CONCLUSION}

The computation of second-order stiffness tensor and an original method to obtain $\gamma$-surfaces in temperature and pressure through $\mathrm{MD}$ simulations allowed us to investigate dislocation core structures in TATB molecular crystal. Elastic constants dependence in pressure shows that anisotropy is not enhanced as the ratio between $C_{i j}$ remains constant as pressure increases. The $\gamma$-surfaces energy landscapes are sligthly smoothed with temperature but preserve their shape between ambient pressure and $10 \mathrm{GPa}$. Dislocations are predicted to have complex core structures within $P 1$ and $P 2$ planes and to spread over approximately a hundred of Burgers vectors $\mathbf{b}$ with a splitting into up to four partials (with a width at halfmaximum around $10 \mathrm{~b}$ ), regardless the plane or the direction. Screw dislocations are approximatively $30 \%$ thinner than edge ones. Thus, dislocation friction on the lattice is unlikely to play a dominant role for the viscoplastic behavior of TATB material for the range of pressures and temperatures explored. The estimation of the maximum von Mises stress along the MEPS at ambient conditions is around $800 \mathrm{MPa}$ that defines an upper bound for the shear stresses within (001) planes.

Second order elastic components for each investigated couple of $(T, P)(\mathrm{PDF})$

\section{AUTHOR INFORMATION}

\section{Corresponding Author}

*E-mail: christophe.denoual@cea.fr.

ORCID

Paul Lafourcade: 0000-0002-4416-6091

Notes

The authors declare no competing financial interest.

\section{REFERENCES}

(1) Cady, H. H.; Larson, A. C. The crystal structure of 1,3,5-triamino2,4,6-trinitrobenzene. Acta Crystallogr. 1965, 18, 485-496.

(2) Bedrov, D.; Borodin, O.; Smith, G. D.; Sewell, T. D.; Dattelbaum, D. M.; Stevens, L. L. A molecular dynamics simulation study of crystalline 1,3,5-triamino-2,4,6-trinitrobenzene as a function of pressure and temperature. J. Chem. Phys. 2009, 131, 224703.

(3) Kroonblawd, M. P.; Sewell, T. D. Theoretical determination of anisotropic thermal conductivity for crystalline 1,3,5-triamino-2,4,6trinitrobenzene (TATB). J. Chem. Phys. 2013, 139, 074503.

(4) Kroonblawd, M. P.; Sewell, T. D. Theoretical determination of anisotropic thermal conductivity for initially defect-free and defective TATB single crystals. J. Chem. Phys. 2014, 141, 184501.

(5) Mathew, N.; Sewell, T. D. Generalized stacking fault energies in the basal plane of triclinic molecular crystal 1,3,5-triamino-2,4,6trinitrobenzene. Philos. Mag. 2015, 95, 424-440.

(6) Mathew, N.; Sewell, T. D. Nanoindentation of the Triclinic Molecular Crystal 1,3,5-Triamino-2,4,6-trinitrobenzene: A Molecular Dynamics Study. J. Phys. Chem. C 2016, 120, 8266-8277.

(7) Kroonblawd, M. P.; Sewell, T. D.; Maillet, J.-B. Characteristics of energy exchange between inter- and intramolecular degrees of freedom in crystalline 1,3,5-triamino-2,4,6-trinitrobenzene (TATB) with implications for coarse-grained simulations of shock waves in polyatomic molecular crystals. J. Chem. Phys. 2016, 144, 064501.

(8) Mallik, B. S.; Kuo, I.-F. W.; Fried, L. E.; Siepmann, J. I. Understanding the solubility of triamino-trinitrobenzene in hydrous tetramethylammonium fluoride: a first principles molecular dynamics simulation study. Phys. Chem. Chem. Phys. 2012, 14, 4884-4890.

(9) Field, J. E. Hot spot ignition mechanisms for explosives. Acc. Chem. Res. 1992, 25, 489-496.

(10) Armstrong, R. W.; Ammon, H. L.; Elban, W. L.; Tsai, D. H. Investigation of hot spot characteristics in energetic crystals. Thermochim. Acta 2002, 384, 303-313. 
(11) An, Q.; Goddard, W. A.; Zybin, S. V.; Jaramillo-Botero, A.; Zhou, T. Highly Shocked Polymer Bonded Explosives at a Nonplanar Interface: Hot-Spot Formation Leading to Detonation. J. Phys. Chem. C 2013, 117, 26551-26561.

(12) Eason, R. M.; Sewell, T. D. Molecular Dynamics Simulations of the Collapse of a Cylindrical Pore in the Energetic Material $\alpha$-RDX. J. Dyn. Behav. Mater. 2015, 1, 423-438.

(13) Austin, R. A.; Barton, N. R.; Reaugh, J. E.; Fried, L. E. Direct numerical simulation of shear localization and decomposition reactions in shock-loaded HMX crystal. J. Appl. Phys. 2015, 117, 185902.

(14) Rai, N.; Bhatt, D.; Siepmann, J. I.; Fried, L. E. Monte Carlo simulations of 1,3,5-triamino-2,4,6-trinitrobenzene (TATB): Pressure and temperature effects for the solid phase and vapor-liquid phase equilibria. J. Chem. Phys. 2008, 129, 194510.

(15) Bulatov, V. V.; Cai, W. Computer simulations of dislocations; Oxford series on materials modelling; Oxford University Press: Oxford, NY, 2006.

(16) Denoual, C. Dynamic dislocation modeling by combining Peierls Nabarro and Galerkin methods. Phys. Rev. B: Condens. Matter Mater. Phys. 2004, 70, 024106.

(17) Denoual, C. Modeling dislocation by coupling Peierls-Nabarro and element-free Galerkin methods. Comp. Met. Appl. Mech. Eng. 2007, 196, 1915-1923.

(18) Metsue, A.; Carrez, P.; Denoual, C.; Mainprice, D.; Cordier, P. Peierls-Nabarro modelling of dislocations in diopside. Phys. Chem. Miner. 2010, 37, 711-720.

(19) Giacomazzi, L.; Carrez, P.; Scandolo, S.; Cordier, P. Dislocation properties of coesite from an ab-initio parametrized interatomic potential. Phys. Rev. B: Condens. Matter Mater. Phys. 2011, 83, 014110.

(20) Peierls, R. The size of a dislocation. Proc. Phys. Soc. 1940, 52, 34.

(21) Cao, L.; Koslowski, M. Rate-limited plastic deformation in nanocrystalline Ni. J. Appl. Phys. 2015, 117, 244301.

(22) Cao, L.; Hunter, A.; Beyerlein, I. J.; Koslowski, M. The role of partial mediated slip during quasi-static deformation of 3D nanocrystalline metals. J. Mech. Phys. Solids 2015, 78, 415-426.

(23) Koslowski, M.; Cuitiño, A. M.; Ortiz, M. A phase-field theory of dislocation dynamics, strain hardening and hysteresis in ductile single crystals. J. Mech. Phys. Solids 2002, 50, 2597-2635.

(24) Soulard, L. Molecular Dynamics Study of the Micro-spallation. Eur. Phys. J. D 2008, 50, 241.

(25) Onsager, L. Electric Moments of Molecules in Liquids. J. Am. Chem. Soc. 1936, 58, 1486-1493.

(26) Barker, J. A.; Watts, R. O. Monte Carlo studies of the dielectric properties of water-like models. Mol. Phys. 1973, 26, 789-792.

(27) Watts, R. O. Monte Carlo studies of liquid water. Mol. Phys. 1974, 28, 1069-1083.

(28) Verlet, L. Computer "Experiments" on Classical Fluids. II. Equilibrium Correlation Functions. Phys. Rev. 1968, 165, 201-214.

(29) Pal, A.; Picu, C. R. Contribution of molecular flexibility to the elastic-plastic properties of molecular crystal ? -RDX. Modell. Simul. Mater. Sci. Eng. 2017, 25, 015006.

(30) Parrinello, M.; Rahman, A. Crystal Structure and Pair Potentials: A Molecular-Dynamics Study. Phys. Rev. Lett. 1980, 45, 1196-1199.

(31) Parrinello, M.; Rahman, A. Polymorphic transitions in single crystals: A new molecular dynamics method. J. Appl. Phys. 1981, 52, 7182-7190.

(32) Frenkel, D.; Smit, B. Understanding Molecular Simulation; Academic Press, Inc., 2001.

(33) Stevens, L. N.; Velisavljevic, D. H.; Dattelbaum, D. Hydrostatic Compression Curve for Triamino-Trinitrobenzene Determined to 13.0 GPa with Powder X-Ray Diffraction. Propellants, Explos., Pyrotech. 2008, 33, 286-295.

(34) Olinger, B.; Cady, H. The Hydrostatic Compression of Explosives and Detonation Products to $10 \mathrm{GPa}$ (100 kbars) and Their Calculated Shock Compression: Results for PETN, TATB, $\mathrm{CO}_{2}$ and $\mathrm{H}_{2} \mathrm{O}$. 6th Symposium (International) on Detonation, Coronado, California, August 24-27, 1976; p 224.

(35) Pineau, N. Cold curve of TATB. 20th Symposium (International) on Detonation, 2015.
(36) Wallace, D. C. Statistical Physics Of Crystals And Liquids; World Scientific, 2002.

(37) Parrinello, M.; Rahman, A. Strain fluctuations and elastic constants. J. Chem. Phys. 1982, 76, 2662-2666.

(38) Weinan, E.; Ren, W.; Vanden-Eijnden, E. String method for the study of rare events. Phys. Rev. B: Condens. Matter Mater. Phys. 2002, 66, 052301.

(39) Birch, F. Finite Elastic Strain of Cubic Crystals. Phys. Rev. 1947, $71,809-824$.

(40) Wallace, D. C. Thermoelasticity of Stressed Materials and Comparison of Various Elastic Constants. Phys. Rev. 1967, 162, 776789.

(41) Hirth, J. P.; Lothe, J. Theory of Dislocations; Krieger Publishing Company, 1982. 